\title{
Cyclic electromechanical response of poly(vinylidene fluoride)
}

\section{Harish* and C. Lakshmana Rao}

Department of Applied Mechanics, Indian Institute of Technology Madras, Chennai - 600036, India Email: harish06117@gmail.com

Email: lakshman@iitm.ac.in

*Corresponding author

\begin{abstract}
In this study, we present the results of cyclic electromechanical experiments conducted on uniaxially stretched poly(vinylidene fluoride) (PVDF) films. The experiments were carried out over a range of applied displacement amplitude ranging from $0.5 \mathrm{~mm}$ to $1.5 \mathrm{~mm}$, superposed on an initial stretch on the test samples. The strains were calculated using non-contact speckle monitoring method. The hysteresis plots of mechanical and electromechanical cyclic responses are presented. Stress relaxation was observed up to $70 \%$ in orthogonal to stretch direction and $16 \%$ in the stretch direction. Observed piezoelectricity along both the directions is reported and discussed in the paper.
\end{abstract}

Keywords: poly(vinylidene fluorid); PVDF; cyclic loading; non-contact speckle method; electromechanical response; piezo sensor.

Reference to this paper should be made as follows: Harish, L. and Rao, C.L. (2019) 'Cyclic electromechanical response of poly(vinylidene fluoride)', Int. J. Materials and Structural Integrity, Vol. 13, Nos. 1/2/3, pp.160-171.

Biographical notes: L. Harish is a Research Scholar at the Department of Applied Mechanics, Indian Institute of Technology Madras, India. He received his Masters in Structural Engineering, from the Department of Civil Engineering at Indian Institute of Technology Hyderabad, India and Bachelor of Technology from JNTU Anantapur, India. His area of interests includes characterisation of the smart materials, sensor development, constitutive modelling of engineering materials, and numerical implementation of the models.

C. Lakshmana Rao is a Professor at the Department of Applied Mechanics, IIT Madras, India. He obtained his BTech and MS from IIT Madras and ScD from Massachusetts Institute of Technology, USA. He teaches and works in the areas of impact mechanics, constitutive modelling of materials, piezoelectric sensing and actuation. He has participated in research and consultancy with several organisations. He has co-authored a book on applied Impact mechanics, modelling of engineering materials, and engineering mechanics - static and dynamics.

This paper is a revised and expanded version of a paper entitled 'Cyclic electromechanical response of PVDF' presented at INCAM-2017, MNNIT, Allahabad, 4-7 July 2017. 


\section{Introduction}

Poly(vinylidene fluoride) (PVDF) is well-known for its strong piezoelectricity. Strong piezoelectricity was first discovered in PVDF by Kawai in 1969. It is capable of exhibiting piezoelectricity both as a sensor (detect electrical voltage when perturbing with mechanical forces) and as an actuator (observe mechanical deformation when perturbing with electrical voltage) (Dargahi et al., 2012). Unlike piezo-ceramics such as lead zirconate titanate (PZT), PVDF is highly flexible (15\% elongation), lightweight $\left(1.87 \mathrm{~g} / \mathrm{m}^{3}\right)$, having good mechanical strength $(60 \mathrm{MPa})$, high conductivity, and charge density (Sathiyanarayan et al., 2006). Due to these properties, PVDF has been used in many applications such as in robotics as tactile sensors, biomedical devices, detecting insect locomotion, customised sensors for structural health monitoring, etc. (Dargahi et al., 2012). Piezoelectricity of PVDF can be realised in many ways (Sokhanvar et al., 2007). In most applications (Vinogradov and Holloway, 1999; Nakahira et al., 2014; Yi and Liang, 2008) PVDF film is subjected to in-plane stretch, and the mechanical and electromechanical response is reported. In many sensor applications (shown in Table 1), the PVDF is being subjected to cyclic loads. The mechanical and electromechanical response of PVDF for cyclic loads is important for assessing the stability of electromechanical properties with repeated use.

Thin films of PVDF $(28 \mu \mathrm{m})$ are often used for sensor applications. These thin films are manufactured with silver ink coating to ensure conductivity on the surface of the film. Thin film sheets are available in two forms:

a biaxially stretched

b uniaxially stretched forms.

Uniaxially stretched films are preferred in many applications for their high strength and electromechanical strain coefficient ( $22 \mathrm{pC} / \mathrm{N}$ in stretched direction) in comparison to biaxially stretched PVDF which has an electromechanical strain coefficient of $5 \mathrm{pC} / \mathrm{N}$. Hence, uniaxially stretched films are taken up for detailed investigation in this study.

Several authors did static mechanical and electromechanical experiments on uniaxially stretched PVDF and have identified strong anisotropy in two in-plane directions (Vinogradov and Holloway, 1999; Harish and Rao, 2017). In these studies, the mechanical and electromechanical properties of uniaxially stretched PVDF were shown to be more in the stretched direction compared to un-stretched direction. Unlike uniaxially stretched PVDF membranes, biaxial membranes show isotropic response in mechanical and electromechanical properties. In this study, we used uniaxial PVDF of $28 \mu \mathrm{m}$ thick membrane samples to conduct cyclic electromechanical experiments.

Strain measurements and calculations in most elastomeric membranes are normally done by using data from crosshead movements. This gives an average strain and is less accurate compared to point strains that are obtained using electrical strain gauges. Strain gauges cannot be used for finite strain measurement in elastomers, since they are normally calibrated for infinitesimal strains. Further, these gauge indicators cannot be easily bonded to thin elastomers. In recent times, non-contact speckle method was used to measure local strains. Harish and Rao (2017) used non-contact speckle monitoring method for measurement of the stress-strain response of PVDF and reported stiffer stress-strain curves compared with those obtained from the crosshead movement for 
monotonic uniaxial and biaxial experiments. Non-contact speckle monitoring method is used for the measurement of cyclic strains in the current study.

Several cyclic experiments of polymers and piezopolymers have been reported recently in the literature. Some of the important works are tabulated in Table 1.

Table 1 Literature on cyclic response of polymers and piezopolymers

\begin{tabular}{ll}
\hline Author & \multicolumn{1}{c}{ Material/strain range/frequency/no. of cycles } \\
\hline $\begin{array}{l}\text { Pieczyska et al. } \\
\text { (2017) }\end{array}$ & $\begin{array}{l}\text { Studied the cyclic response of shape memory polymers to characterise } \\
\text { the yielding phenomena by numerical technique. }\end{array}$ \\
Chen et al. (2016) & $\begin{array}{l}\text { Applied periodic cyclic loads by vibration exciter to investigate the } \\
\text { response of PVDF-TrFE with nanofillers and plotted voltage Vs. Time } \\
\text { for the different percentage of fillers and size of the nanowires. With } \\
\text { fillers and particular size, PVDF-TrFE sensitivity response improved by } \\
\text { factor three. }\end{array}$ \\
Kim et al. (2003) & $\begin{array}{l}\text { Found a method for estimating the weight of moving vehicles using } \\
\text { piezo sensors. }\end{array}$ \\
Moon et al. (2007) & $\begin{array}{l}\text { Studies tire/road interactions using PVDF as deformation sensor and } \\
\text { energy harvester. }\end{array}$ \\
Lee et al. (2007) & $\begin{array}{l}\text { Used PVDF sensor to monitor cockroach locomotion which is subjected } \\
\text { to cyclic deformation. }\end{array}$ \\
Shen et al. (2004) & $\begin{array}{l}\text { Investigated the cyclic behaviour of epoxy polymer. In strain controlled } \\
\text { cyclic loading, the epoxy polymer was amplitude dependent, got } \\
\text { cracked at 70 cycles for 3\% strain, 600 cycles for 1.5\% strain and mean } \\
\text { stress relaxation was observed. } \\
\text { The cyclic stress-strain behaviour of different rigid polymers. }\end{array}$ \\
\hline Rabinowitz and &
\end{tabular}

From the above literature (shown in Table 1) we can understand that most polymers are tested for a low number of cycles, while intended purpose is for sensors, which are expected to be used at least for about 1,000 cycles. Measurement of strains in all the studies is made from cross-head movement while such measures do not give accurate point measurement of strain and are inferior to non-contact speckle methods. PVDF as a sensor is also likely to be used in cyclic applications and the stability of its mechanical and electromechanical properties after repeated use are not quantified by other researchers.

Hence the objectives of the paper are:

a to test the uniaxial PVDF films under cyclic loading conditions for 1,000 cycles

b to use non-contact speckle monitoring method is used to find point strains

c to assess the stability of mechanical and electromechanical response of the PVDF after application of cyclic loads.

Section 2 of the paper will discuss the detail test procedure and sample made from sheets. Section 3 and its subsection of the article will present the results of the variation of the response of the amplitude, hysteresis variation response with a number of cycles. 


\section{Material and test method}

Uniaxially stretched PVDF sheet purchased from Measurement Specialities (MEAS Inc.) has the dimensions $10 \times 13 \mathrm{~cm}$ as shown in Figure 1(a). The sheet has polling of stretch direction in direction 1 and is cut into filmstrips (of dimension $7.5 \mathrm{~cm} \times 1.5 \mathrm{~cm}$ ) along the direction 1 and direction 2 . The filmstrips have a silver ink coating on top and bottom, for good conductivity on the surface of the filmstrips.

Figure 1 (a) PVDF sheet and assigned directions (b) Cut sample of PVDF (see online version for colours)

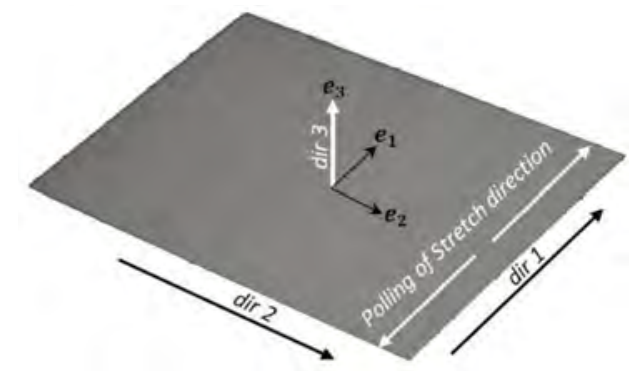

(a)

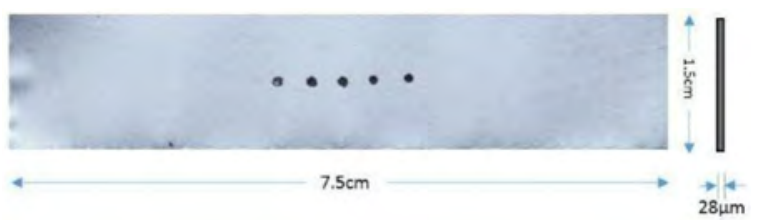

(b)

Figure 2 (a) Biaxial test setup (b) Schematic view (see online version for colours)

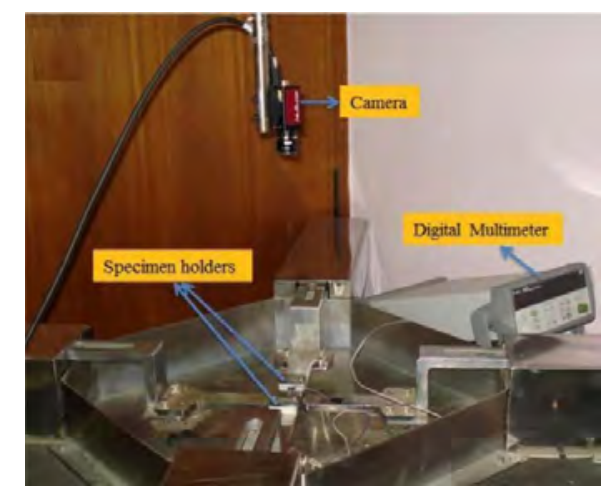

(a)

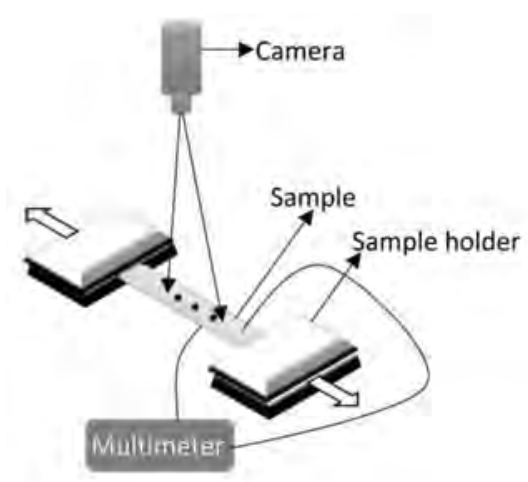

(b)

A biaxial stretching machine (schematically shown in Figure 2) was used to conduct the experiments. The test setup can conduct experiments for thin films, loading range up to $1 \mathrm{kN}$. After cutting the sample to the proposed dimension, the sample gets a short circuit 
and would not get voltage. The methanol solution was used to remove the coating on the edges of the film to avoid the short circuit. A digital multimeter (Agilent 34972A) was used to measure the voltage variation in the film while mechanically stimulating the PVDF film.

Table 2 Applied amplitudes for cyclic experiments

\begin{tabular}{lcc}
\hline Experiment number & Direction-1 amplitude & Direction-2 amplitude \\
\hline 1 & $0.5 \mathrm{~mm}$ & $0.5 \mathrm{~mm}$ \\
2 & $0.75 \mathrm{~mm}$ & $1 \mathrm{~mm}$ \\
3 & $1 \mathrm{~mm}$ & $1.5 \mathrm{~mm}$ \\
\hline
\end{tabular}

A displacement control mode option was used for biaxial test setup to conduct cyclic experiments. Centring of the film was done and $1 \mathrm{~mm}$ pre-stretch was given on both sides of the film before conducting the experiment. Due to high anisotropy of the uniaxially stretched PVDF film, we have used the different strain ranges to conduct the cyclic experiment; this ensures that the material is in elastic range and does not wrinkle during the experiment. Cyclic cross-head displacement amplitudes of $0.5 \mathrm{~mm}$ to $1.5 \mathrm{~mm}$ were given in direction 1 and direction 2 respectively, as indicated in Table 2. Experiments were conducted at constant frequency of $1 \mathrm{~Hz}$. To find strains locally on the film, a non-contact speckle monitoring method was used. Black speckle marks were applied on the film. A low-speed high-resolution camera (Stingray F125 C) was used to capture the film image while conducting the experiment. MATLAB R2016a (9.0.0.341360) code and image viewer tools were used to find the strains from the raw images. Image grabbing speed of 7.5 frames per second (FPS) was used to capture the images. To synchronise the data, we ensured that the same frames per second was used in Agilent multimeter and the camera attached to biaxial test setup as well.

\section{Results and discussion}

The results of mechanical and electromechanical cyclic experiments conducted as per the experimental procedures outlined in Section 3 are reported in two in-plane directions (stretch direction and orthogonal to stretch direction) for 1,000 cycles. Variation of stress-strain hysteresis plots, voltage and stress plots with respect to number of cycles, are presented and discussed in detail in the subsections, listed below.

\subsection{Variation of response amplitude with number of cycles}

Cyclic response experiments under displacement-controlled loading on PVDF samples were conducted. All tests were conducted at high strain ranges (up to 10\%). The variation of stress and voltage with time for different amplitudes in two in-plane directions are plotted in Figures 3 and 4. The nominal stress points are plotted on the left Y-axis, and voltage points plotted on the secondary right $\mathrm{Y}$-axis. Time is common axis for both the $\mathrm{Y}$-axes. The mean stress and voltage values are plotted with number of cycles in stretch direction are showing in Figure 5 (each point in $\mathrm{X}$-axis represents the mean of two cycles). Since nominal stress (based on original area of cross-section), strain (from image), voltage (from Agilent meter) data points measured at 7.5 readings/frames per sec, 
each of X-axis represents $0.14 \mathrm{sec}$. Represented stress-voltage variations are shown for 5 th, 500th and 1,000th cycles. The displacement amplitude which we applied on stretch direction is different from the amplitude orthogonal to stretch direction (shown in Table 2) since the stiffness of the material is different in the two directions.

Figure 3 Strain-voltage variations concerning time in stretch direction (direction 1) for (a) $0.5 \mathrm{~mm}$ amplitude, (b) $1 \mathrm{~mm}$ amplitude and (c) $1.5 \mathrm{~mm}$ amplitude (see online version for colours)

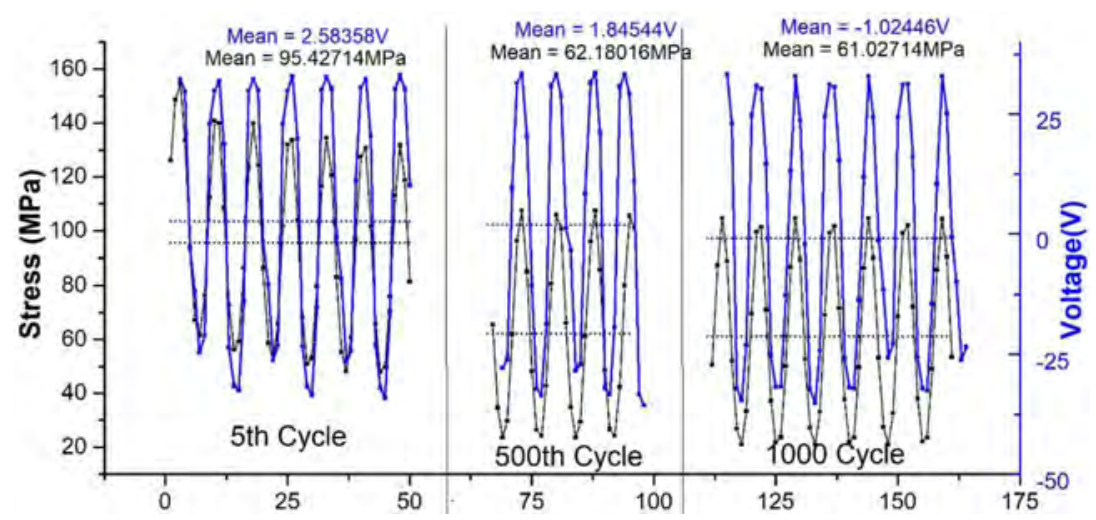

(a)

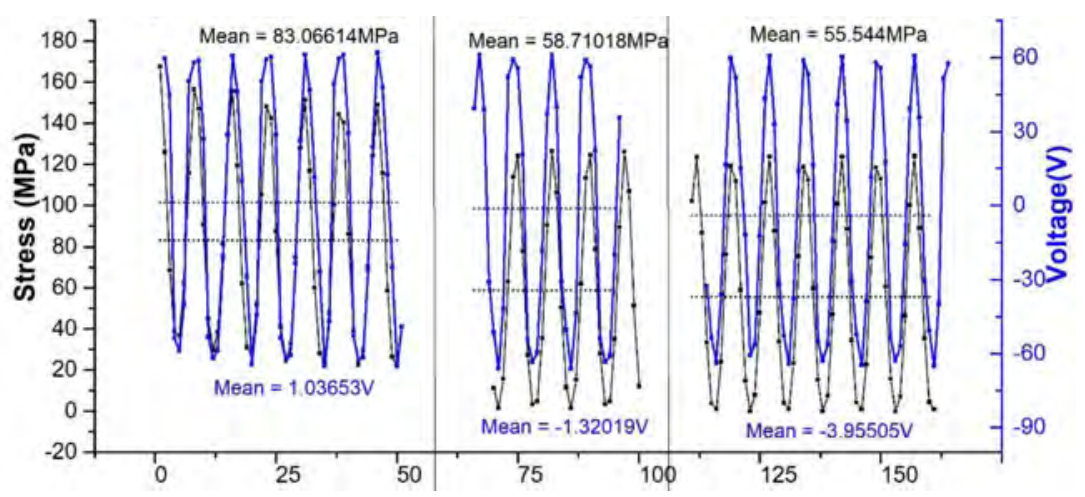

(b)

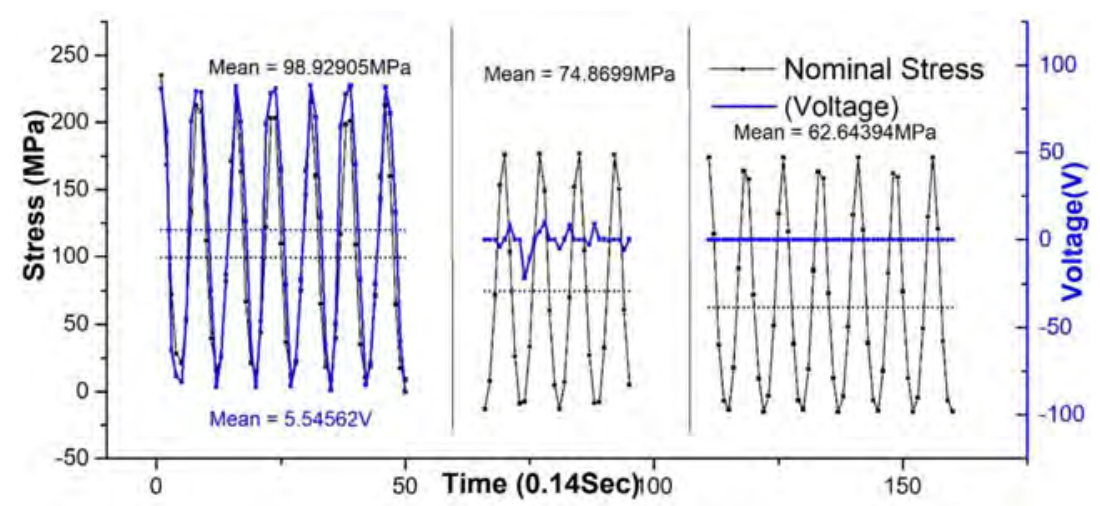

(c) 
Figure 4 Strain-voltage variations concerning time in orthogonal to stretch direction (direction 2) for (a) $0.5 \mathrm{~mm}$ amplitude, (b) $0.75 \mathrm{~mm}$ amplitude and (c) $1 \mathrm{~mm}$ amplitude (see online version for colours)

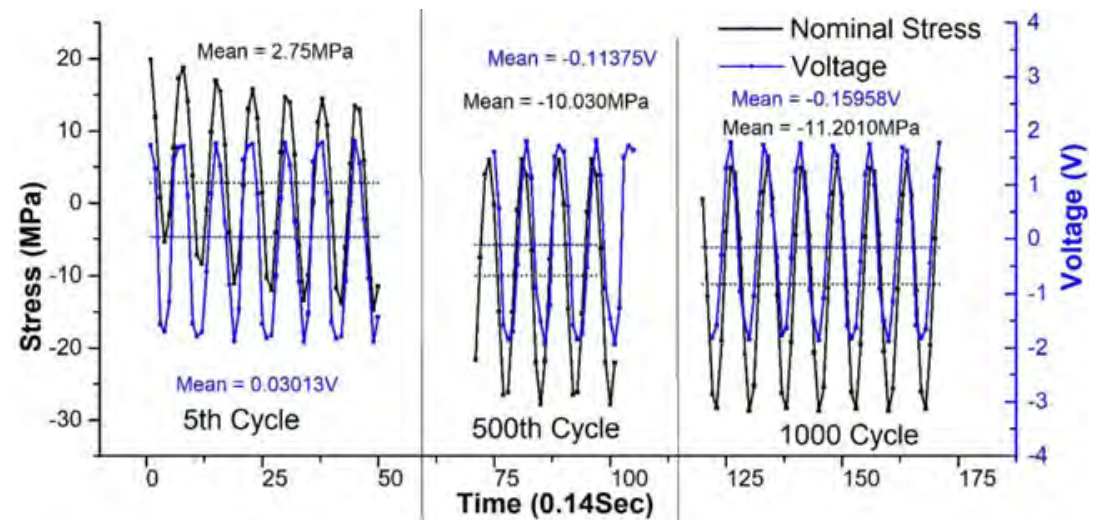

(a)

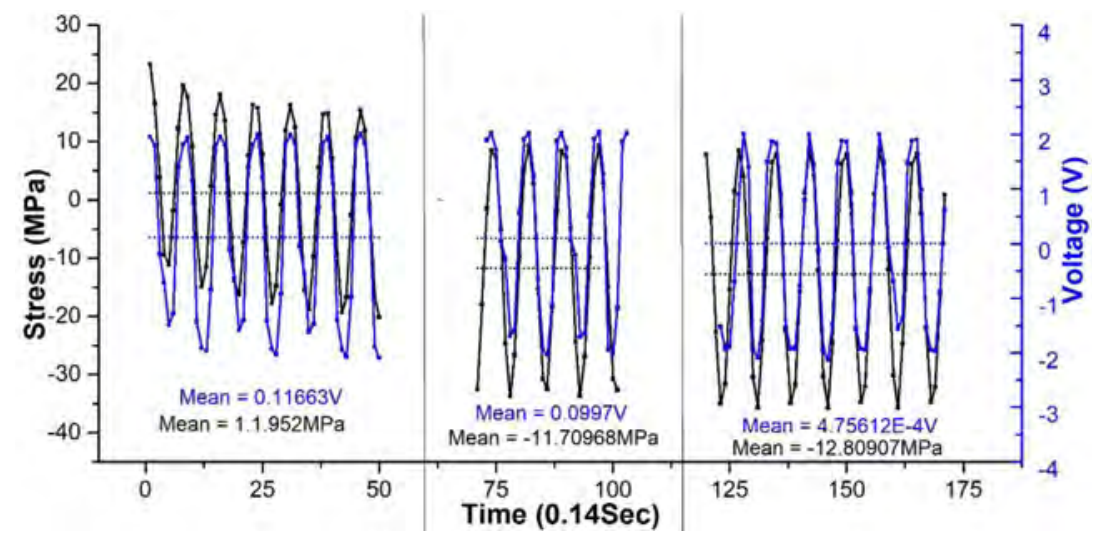

(b)

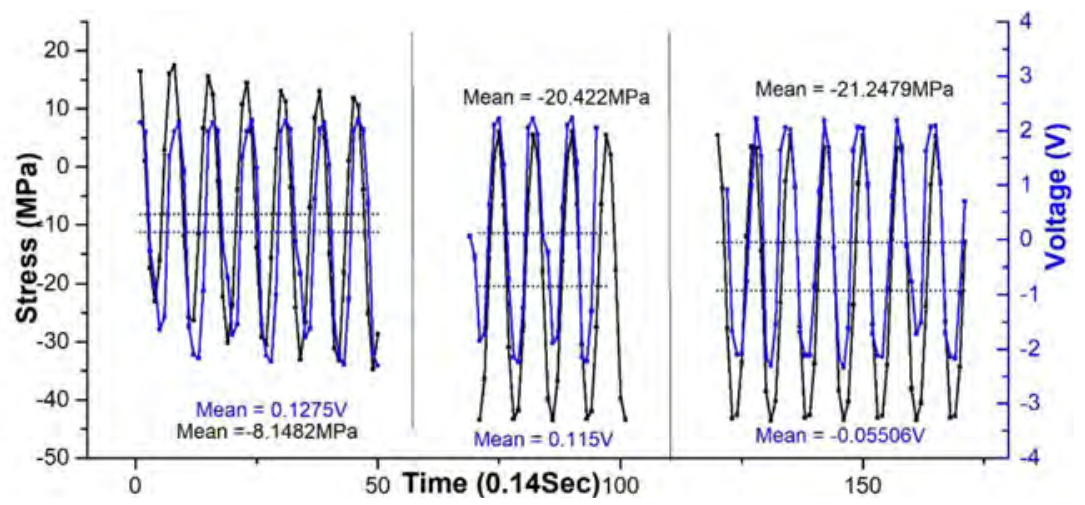

(c) 
Figure 5 (a) Mean stress and (b) mean voltage variation with number of cycles in stretch direction of the PVDF (see online version for colours)

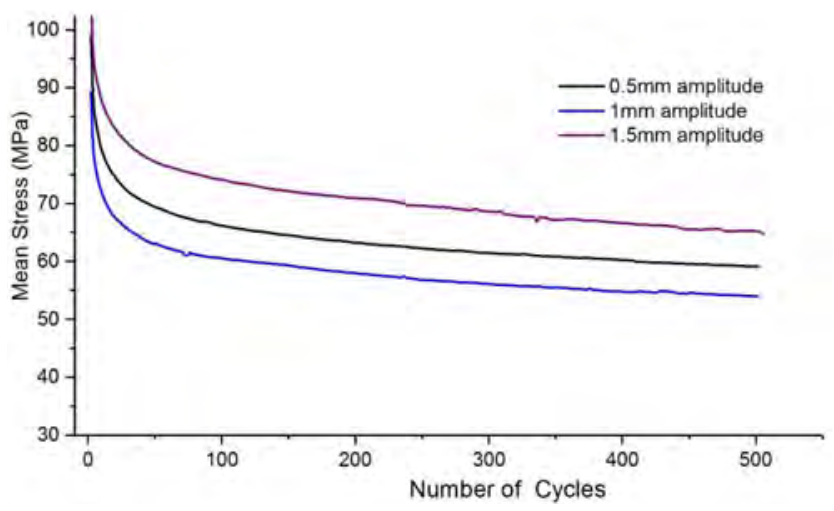

(a)

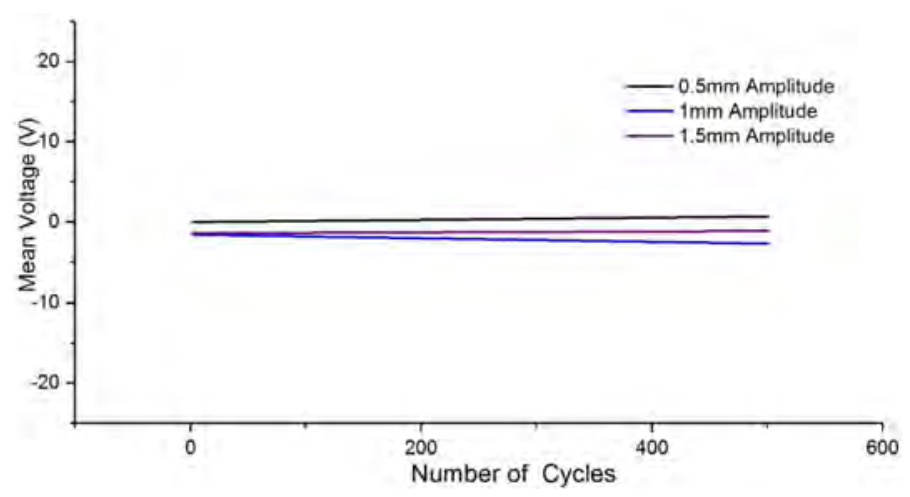

(b)

The mean value line is shown in the plots (Figure 3, 4 and 5) for stress and voltage. It may be observed that mean value decreases with number of cycles and high stress drop can be observed in first 100 cycles. Due to semicrystallinity in stretch direction and semi-amorphous, the mean stress drop is high in first few cycles than the later cycles. We notice the same pattern of change in all amplitudes of stresses in both the stretch direction and orthogonal to the stretch direction. Voltage is showing steadily the same across all cycles in both the directions irrespective of mean stress variation. Amplitude of voltage can be observed to be less in orthogonal to stretch direction whereas it about 10 times higher in the stretch direction. Even though the film did not exhibit any damage mechanically in stretch direction, a significant voltage drop has observed around 333rd cycles at $1.5 \mathrm{~mm}$ amplitude as shown in Figure 3(c). This may be due to the disturbance of electrical polling. In orthogonal to stretch direction, the material has yielded in initial cycle itself at $1 \mathrm{~mm}$ amplitude. 


\subsection{Variation of hysteresis response with number of cycles}

Stress-strain hysteresis curves are plotted for 1st, 500th, and 1,000th cycles for different amplitudes, for the two in-plane directions. The plots are shown in Figures 6 and 7. It may be observed that the hysteresis loops are generally elliptical in shape. The centre of these ellipses indicates the mean state of stress/strain in the material and the orientation of the major axis indicates the average stiffness in the material at that stage of stress. The area of the ellipse indicates the dissipation due to cyclic loading in the material.

Figure 6 Stress-strain plots in stretch direction for (a) $0.5 \mathrm{~mm}$ amplitude, (b) $1 \mathrm{~mm}$ amplitude and (c) $1.5 \mathrm{~mm}$ amplitude (see online version for colours)

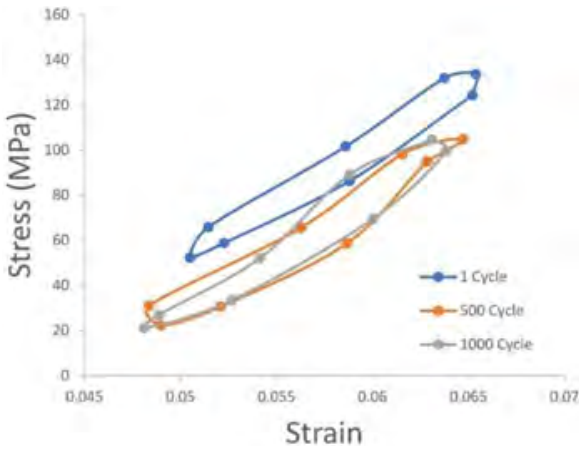

(a)

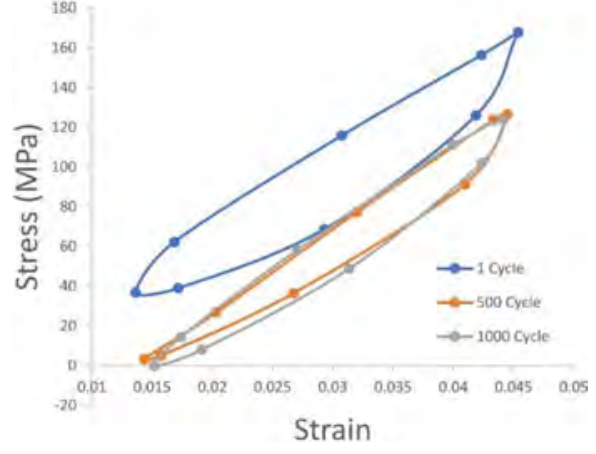

(b)

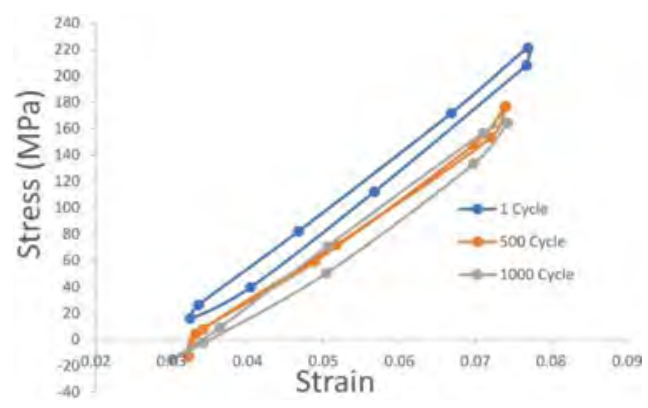

(c)

It may be observed from the figures that the hysteresis loop is increasing with increasing number of cycles and also with increase in amplitude of loading. In stretch direction, loading with $1 \mathrm{~mm}$ amplitude resulted in higher hysteresis compared to loading with $0.5 \mathrm{~mm}$ and $1.5 \mathrm{~mm}$ amplitude. Due to the early yield observation at $1 \mathrm{~mm}$ amplitude, we can see more energy dissipation from Figure $7(\mathrm{c})$. About $70 \%$ stress relaxation is observed from initial to the last cycle in orthogonal to stretch direction whereas $16 \%$ stress relaxation seen in stretch direction. By comparing the stress in the two orthogonal axes, it may be noticed that the strength of the PVDF is high in stretched direction compared to orthogonal to stretch direction. The induced stress amplitudes are also more in stretch direction compared to orthogonal direction. The slopes of the graphs seem consistent irrespective of the amplitude and number of cycles. Dissipation levels in the material seem to increase with amplitude of displacement. Due to the better properties observed in stretch direction and poor electromechanical response in the orthogonal 
direction, only voltage vs. strain plots along the stretch direction are shown in Figure 8. It can be observed from Figure 8 that, the amplitude of voltage and slope is consistent with number of cycles and displacement amplitude. The slope of the hysteresis seems the same in all amplitudes and mean state of the voltage is not changing for $1 \mathrm{~mm}$ amplitude case. Zero hysteresis can be observed due to the sudden drop of voltage at $1.5 \mathrm{~mm}$ amplitude.

Figure 7 Stress-strain plots in orthogonal to stretch direction for (a) $0.5 \mathrm{~mm}$ amplitude, (b) $0.75 \mathrm{~mm}$ amplitude and (c) $1 \mathrm{~mm}$ amplitude (see online version for colours)

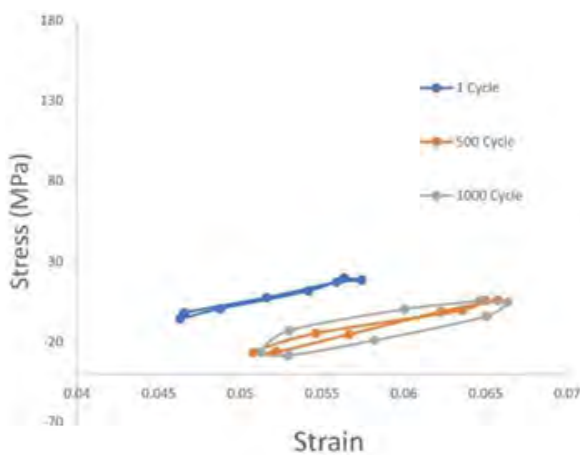

(a)

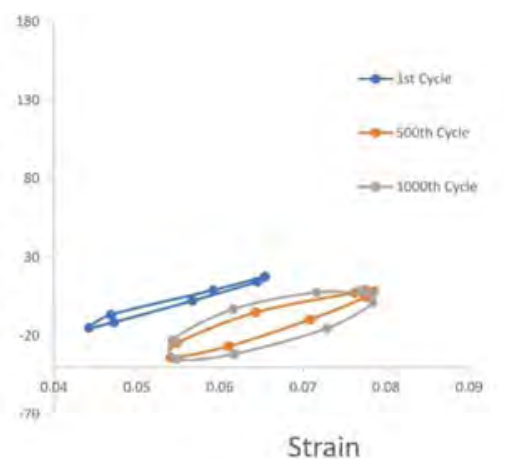

(b)

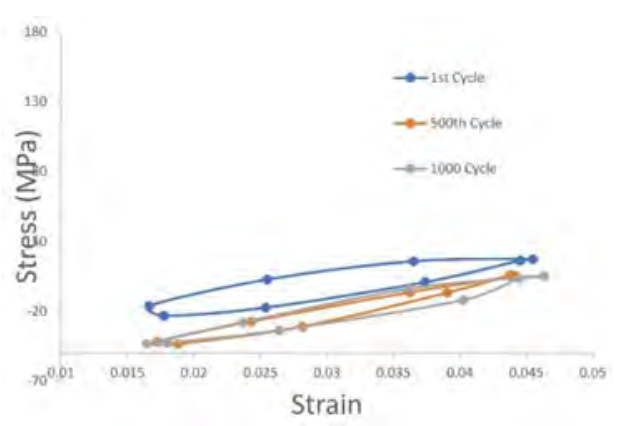

(c)

Figure 8 Voltage vs strain plots in stretch direction for (a) $0.5 \mathrm{~mm}$ amplitude, (b) $1 \mathrm{~mm}$ amplitude and (c) $1.5 \mathrm{~mm}$ amplitude (see online version for colours)

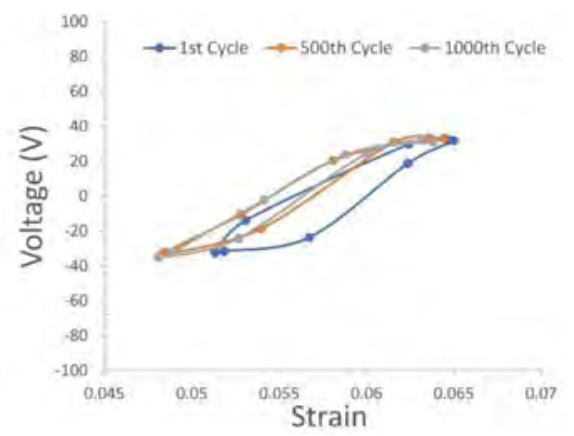

(a)

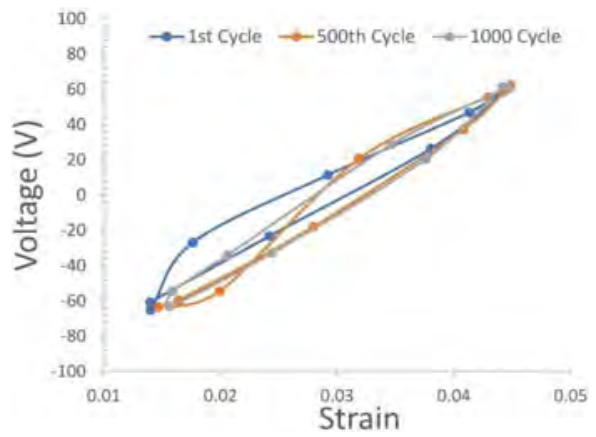

(b) 
Figure 8 Voltage vs strain plots in stretch direction for (a) $0.5 \mathrm{~mm}$ amplitude, (b) $1 \mathrm{~mm}$ amplitude and (c) $1.5 \mathrm{~mm}$ amplitude (continued) (see online version for colours)

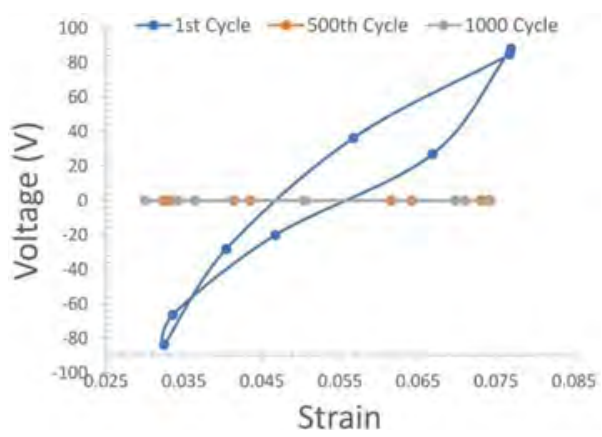

(c)

\section{Summary and conclusions}

In this study, cyclic electromechanical experiments were conducted on uniaxially stretched PVDF for 1,000 cycles in two directions. Strains were measured using non-contact speckle monitoring method. The following significant observations may be observed from the experimental studies:

a The mechanical as well as the electromechanical response in the stretch direction, is more than 10 times higher than the equivalent response in the orthogonal direction. Hence, these membranes must be considered for sensing applications only along the stretch direction. The higher properties in the stretch direction are due to semi-crystalline of polymer and mechanical stretch during the making of PVDF.

b There is about $30 \%$ drop in the mean value of the mechanical response within the first 100 cycles, in the stretch direction. The drop in the mechanical response in the next 100 cycles is insignificant. Similar trends were also observed in the orthogonal direction. Hence, it may be concluded that these membranes can be used effectively within about 100 cycles and with reduced sensitivity for higher number cycles. The mean drop in the first cycles may be due to the semi-amorphous and crystalline nature of the PVDF.

c The mean value of electromechanical response along the stretch direction is about $30 \%$ in the first 100 cycles for low amplitudes of displacement. This decrease is further amplified at higher amplitudes of displacement. One can also observe a general trend of a decrease in the mean response with an increase in the number of cycles at lower displacement amplitudes. The results seem to indicate a breakdown of electromechanical activity at higher amplitudes $(1.5 \mathrm{~mm})$ and a higher number of cycles ( $>300$ cycles). The voltage drop in the polymer may be due to the disturbance of electrical polling in the thickness direction.

d The average slope stress-strain response is not changing with a number of cycles or displacement amplitudes along both the directions of the sample. Wherever the electromechanical response exists, the slope of the voltage - strain plot also does not change with a number of cycles. 
The energy dissipation (as observed in the area of stress-strain plots) is increasing for first 100 cycles and remains the same for later cycles. The hysteresis in the voltage strain plots (where they exist) is similar to the stress hysteresis. In general, the experimental results seem to indicate that the mechanical and electromechanical properties of PVDF are more stable within the first 300 cycles and for lower amplitudes of displacement loading. Hence, these membranes are to be used only in the stretch direction within these ranges of amplitudes and number of cycles. Hence, attempts to model this response should be made only within these ranges. This will be attempted in future work by the authors.

\section{References}

Chen, H-J., Han, S., Liu, C., Luo, Z., Shieh, H-P.D., Hsiao, R-S., and Yang, B-R. (2016) 'Investigation of PVDF-TrFE composite with nanofillers for sensitivity improvement', Sensors and Actuators A: Physical, Vol. 245, pp.135-139.

Dargahi, J. et al. (2012) Tactile Sensing and Display: Haptic Feedback for Minimally Invasive Surgery and Robotics, John Wiley \& Sons.

Harish, L. and Rao, C.L. (2017) 'Characterization of the monotonic uniaxial and biaxial mechanical response of polyvinylidene fluoride (PVDF) films', Experimental Techniques, Vol. 41, No. 4, pp.1-7.

Kawai, H. (1969) 'The piezoelectricity of poly (vinylidene fluoride)', Japanese Journal of Applied Physics, Vol. 8, No. 7, p.975.

Kim, S-W, Cho, I., Lee, J-H., Park, J., Yi, D-H. and Cho, D.D. (2003) 'A new method for accurately estimating the weight of moving vehicles using piezoelectric sensors and adaptive-footprint tire model', Vehicle System Dynamics, Vol. 39, No. 2, pp.135-148.

Lee, H., Cooper, R., Mika, B., Clayton, D., Garg, R., Gonzalez, J.M., Vinson, S.B., Khatri, S. and Liang, H. (2007) 'Polymeric sensors to monitor cockroach locomotion', IEEE Sensors Journal, Vol. 7, No. 12, pp.1698-1702.

Moon, K.S. et al. (2007) 'Tire tread deformation sensor and energy harvester development for smart-tire applications', Sensors and Smart Structures Technologies for Civil, Mechanical, and Aerospace Systems 2007, Vol. 6529, International Society for Optics and Photonics.

Nakahira, K., Tago, H., Sasaki, T., Suzuki, K. and Miura, H. (2014) 'Measurement of the local residual stress between fine metallic bumps in 3D flip chip structures', International Journal of Materials and Structural Integrity, Vol. 8, Nos. 1-3, pp.21-31.

Pieczyska, E.A., Staszczak, M., Kowalczyk-Gajewska, K., Maj, M., Golasiński, K., Golba, S., Tobushi, H. and Hayashi, S. (2017) 'Experimental and numerical investigation of yielding phenomena in a shape memory polymer subjected to cyclic tension at various strain rates', Polymer Testing, Vol. 60, pp.333-342.

Rabinowitz, S. and Beardmore, P. (1974) 'Cyclic deformation and fracture of polymers', Journal of Materials Science, Vol. 9, No. 1, pp.81-99.

Sathiyanarayan, S., Sivakumar, S.M. and Rao, C.L. (2006) 'Nonlinear and time-dependent electromechanical behavior of polyvinylidene fluoride', Smart Materials and Structures, Vol. 15, No. 3, p.767.

Shen, X., Xia, Z. and Ellyin, F. (2004) 'Cyclic deformation behavior of an epoxy polymer. Part I: experimental investigation', Polymer Engineering \& Science, Vol. 44, No. 12, pp.2240-2246.

Sokhanvar, S., Zabihollah, A. and Sedaghati, R. (2007) 'Investigating the effect of the orthotropic property of piezoelectric PVDF', Transactions of the Canadian Society for Mechanical Engineering, Vol. 31, No. 1, pp.111-125.

Vinogradov, A. and Holloway, F. (1999) 'Electro-mechanical properties of the piezoelectric polymer PVDF', Ferroelectrics, Vol. 226, No. 1, pp.169-181.

Yi, J. and Liang, H. (2008) 'A PVDF-based deformation and motion sensor: modeling and experiments', IEEE Sensors Journal, Vol. 8, No. 4, pp.384-391. 